\title{
High-rate, Full-Diversity STBCs from Field Extensions
}

\author{
V.Shashidhar, K.Subrahmanyam \\ Department of ECE, \\ Indian Institute of Science \\ Bangalore-560012, INDIA \\ subru@protocolece.iisc.ernet.in \\ R.Chandrasekharan, B.Sundar Rajan ${ }^{1}$ \\ Department of ECE, \\ Indian Institute of Science \\ Bangalore-560012, INDIA \\ rchandru@protocol.ece.iisc.ernet. in
}
B.A.Sethuraman
Dept. of Mathematics
California State University, Northridge
CA 91330, USA
al. sethuraman@csun.edu

Abstract - We present a construction of high-rate codes from field extensions that are full-rank also. Also, we discuss the coding gain and decoding of these codes.

\section{High-RATE FULL-DIVERSITY CODES}

Let $\mathbb{Q}$ denote the field of rational numbers and let $S$ be the signal set, over which we want rate $R$ (complex symbols/channel use) Space-Time Block Codes (STBCs) for $n$ transmit antennas. An $n \times n$ STBC is said to be over $S$ if all the codeword matrices have entries that are complex linear combinations of elements of $S$. Consider the following chain of field extensions:

$$
\mathbb{Q} \subset \underbrace{\mathbb{Q}(S)}_{L} \subset \underbrace{\mathbb{Q}(S, \alpha)}_{F} \subset \underbrace{\mathbb{Q}(S, \alpha, \beta)}_{K}
$$

with $[F: L]=R$ and $[K: F]=n$. Then we have Theorem 1 the proof of which is an extension of the proofs given in $[1,2]$ for similar theorems:

Theorem 1 The set of matrices of the form $f_{0}(\alpha) I+$ $f_{1}(\alpha) M_{\beta}+f_{2}(\alpha) M_{\beta}^{2}+\ldots+f_{n-1}(\alpha) M_{\beta}^{n-1}$ where $M_{\beta}$ is the companion matrix of $\beta$ over $F$ and $f_{i}(\alpha)$ is a $R-1$-th degree polynomial in $\alpha$ with coefficients from $L$, has the property that the difference of any two distinct matrices in the set is of full rank and hence, constitutes a rate- $R$, full-rank $S T B C$ over $S$ if the coefficients of $f_{i}(\alpha)$ are restricted to $S \subset L$.

In particular, if the minimal polynomial of $\beta \in K$ over $F$ is of the form $x^{n}-\gamma$, then the codeword matrices are of the form

$$
\left[\begin{array}{cccc}
f_{0}(\alpha) & \gamma f_{n-1}(\alpha) & \cdots & \gamma f_{1}(\alpha) \\
f_{1}(\alpha) & f_{0}(\alpha) & \cdots & \gamma f_{2}(\alpha) \\
\vdots & \vdots & \ddots & \vdots \\
f_{n-1}(\alpha) & f_{n-2}(\alpha) & \cdots & f_{0}(\alpha)
\end{array}\right]
$$

where $f_{i}(\alpha)=\sum_{j=0}^{R-1} f_{i, j} \alpha^{j}$ with $f_{i, j} \in S$. In the above development, the selection of $\alpha$ and $\beta$ depends on the signal set, $R$ and $n$, which restricts the coding gain of the STBC. By choosing $\alpha$ to be a transcendental element over $Q(S)$, the polynomials $f_{i}(\alpha)$, can be of arbitrary degree and hence we get a rate- $R$, full-rank STBC for any arbitrary $R \geq 1$.

\section{CODING GAIN}

Given any field extension $K / F$, let $N_{K / F}(k)$ denote the algebraic norm from $K$ to $F$ of $k \in K$. Then, we have the following theorem:

Theorem 2 Let $K=F(\beta)$ be an $n$-th degree extension of $F$. The coding gain of the $S T B C$ over $S$ constructed as in Theorem 1 is given by $\min _{k \neq k^{\prime}}\left|N_{K / F}\left(k-k^{\prime}\right)\right|^{2 / n}$, where $k=$ $f_{0}+f_{1} \beta+\cdots+f_{n-1} \beta^{n-1}$ and $k^{\prime}=f_{0}^{\prime}+f_{1}^{\prime} \beta+\cdots+f_{n-1}^{\prime} \beta^{n-1}$.

\footnotetext{
${ }^{1}$ This work was partly funded by the DRDO-IISc Program on
} Mathematical Engineering through a grant to B.S.Rajan.
Theorem 3 Let $S$ be a signal set carved out from $\mathbb{Z}[j]$. Let $K$ and $F$ be as in Theorem 2 , with $\beta$ integral over $F$, i.e., minimal polynomial of $\beta$ over $F$ is in $\mathbb{Z}[j][x]$. Then the coding gain of $S T B C$ s constructed as in Theorem 1 with $R=1$, is (i) 1 if $S$ is contains two nearest neighbors of $\mathbb{Z}[j]$ (ii) $4 d^{2}$ if $S$ is a possibly rotated regular $Q A M$ signal set $\left\{\left((2 k-1-Q) d+j\left(2 l-1-Q^{\prime}\right) d\right) e^{j \phi} \mid k \in[1, Q], l \in\left[1, Q^{\prime}\right]\right\}$ for any $\phi \in[0,2 \pi]$.

\section{Decoding and Simulations}

The STBC's constructed in Theorem 1 are sphere decodable when $S$ is a lattice constellation or a PSK signal set. We present simulation results for several such STBCs over QAMsignal sets with $R=1$ obtained with the monic irreducible polynomials, $x^{3}-1-j, x^{4}-j, x^{5}-1-j, x^{6}-1-j, x^{7}-1-j, x^{8}-j$ respectively for $3,4,5,6,7$ and 8 transmit antennas in Figure 1 . The number of receive antennas for 3 and 4 antennas is 2 and for the rest it is 1 . We use sphere decoding to decode our STBCs. The curves match with those given in [3] in all the cases which means these codes are of maximum coding gain.

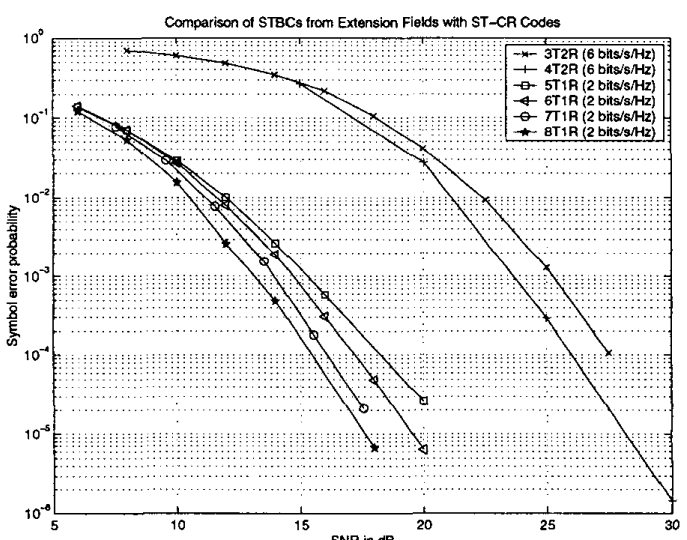

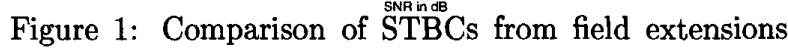
with ST-CR Codes of [3]

\section{REFERENCES}

[1] B. A. Sethuraman and B. Sundar Rajan, "Optimal STBC over PSK signal sets from cyclotomic field extensions," Proc. IEEE ICC 2002, April 28-May2, New York City, USA, Vol.3, pp.17831787.

[2] B. A. Sethuraman and B. Sundar Rajan, "STBC from field extensions of the Rational field," Proc. IEEE ISIT 2002, Switzerland, June 30-July 5, p.274.

[3] Y.Xin, Z.Wang and G.B.Giannakis, "Space-Time ConstellationRotating Codes maximizing diversity and coding gains", Proc. of GLOBECOM, vol.1, pp.455-459, November, 2001. 